\title{
Can RNAi be used as a weapon against COVID-19/SARS- CoV-2?
}

Kawalpreet K Aneja ${ }^{1 *}$, Niketa Dixit ${ }^{2}$ and Arun Kumar ${ }^{3}$

*Correspondence: kaneja@hotmail.com

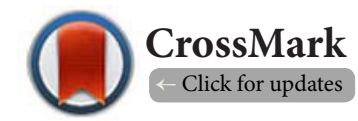

'Independent Scholar, Previous Research Fellow UPENN, Biology Educator at Randolph Career and Technical Institute, Philadelphia 19104, USA.

${ }^{2}$ Research Assistant, Bioprosthetics Inc, Newark DE 19703, USA.

${ }^{3}$ Chief scientist, Bioprosthetics Inc, Newark DE 19703, USA.

\begin{abstract}
Can RNA interference be used as a diagnostic and therapeutic for COVID-19? Can host or viral encoded miRNA or siRNA be used as a vaccine against SARS-CoV-2? RNAi has been used as a platform to make attenuated viral vaccines where the viral genome is engineered and modified to contain miRNA or siRNA binding sites [50]. One of these examples was the creation of self-attenuating Influenza A virus strain that expressed an siRNA from the NS segment (for wild type nonstructural protein NS1) that targets the ORF of the nucleoprotein [NP] segment just at a single site [51]. Intranasal administration of five chemically modified miRNA mimics corresponding to highly expressed miRNAs in respiratory epithelial cells synergistically suppressed H1N1 replication in mice. MicroRNA 122 is another most common example in RNAi literature, antimiR against mir-122 is effective to lower the hepatitis $\mathrm{C}$ virus and miR-122 inhibition by anti-miR122 also reduces serum cholesterol levels [40,52]. RNAi patents and clinical trials for liver cancer, breast cancer, lymphoma, melanoma, IAV, are just a few examples and there are hopes for COVID-19 soon. How about the plant-based diet and plant microRNAs, can we use them against COVID-19 like infections? This mini-review discusses different types of non-coding small RNA molecules, their biogenesis, the role of innate immune response and the competition of proviral and antiviral proteins, and how RNAi can be alone or as a combination of COVID-19 intervention drugs and vaccines used as a therapeutic along with a little emphasis on plant-based miRNAs to prevent future pandemics like COVID-19?
\end{abstract}

Keywords: RNAi, Non-Coding Small RNAs, miRNA, siRNA, Drosha, Dicer, Argonaute Complex, COVID-19, Pattern Recognition Receptors, Interferon, IFN-regulatory factors, IFN-stimulatory genes, Cytokine storm, Vaccine, Plant miRNA

\section{Introduction}

RNAi means active cell response to foreign RNA [1]. RNAi is a major defense mechanism against alien nucleic acids of bacteria, viruses, and phages; RNAi is present in diverse organisms like fungi, plants, algae [2], invertebrates, and vertebrates including mammals. RNAi is known for at least the last two decades, is a double-stranded short interfering RNA (siRNA) or microRNA (miRNA) of approximately 21-23 nucleotides that can target the degradation or prevent the translation of an mRNA which contains complementarity to it [3].

\section{Review}

What is RNA Interference?

RNA interference(RNAi) is not interference but is a process where noncoding RNA regulates gene expression, protein expression, RNA splicing, RNA silencing, chromatin structure, modifications, and segregation [3]. RNAi plays a big role in various biological phenomena like cell proliferation, cell death, fat metabolism in flies, neuronal patterning in nematodes, regulation of hematopoietic cells, and differentiation in mammals, root, stem, and leaves formation in plants [4], etc.

What are the different types of Small RNA Molecules? Based upon the whole genome and transcriptome sequencing projects, and RNAi experiments, there are three main types of small RNAs: microRNA or miRNA, small interfering RNA or siRNA, \& piwi-associated RNA or piRNA.

1.miRNA: They are 20-25 nucleotides long. Gene regulatory 
Aneja et al. Microbiology Discovery 2020,

http://www.hoajonline.com/journals/pdf/2052-6180-8-1.pdf

doi: $10.7243 / 2052-6180-8-1$

network depicts miRNAs as endogenous, processed from larger dsRNA precursors,imperfect stem-loop structures, or partially double-stranded structures $[3,5,6]$. The miRNAs bind mainly 3'UTR by Watson-Crick base pairing; the region 2-3 bases 5 'of miRNA that has exact complementarity are the seed sequence, and binding of miRNA results in a translational arrest, deadenylation, and degradation of the transcript.

2.siRNA: These were considered primarily exogenous in origin but recently endogenous siRNAs have been discovered $[6,18]$. ExogenoussiRNAs are 20-25 nucleotides long, their main goal is to defend the genomic integrity; they are derived directly from viruses, transposons, repeat-associated transcripts, centromere [3], or by transgene trigger; and are excised from long, fully complementary double-stranded RNAs. EndosiRNAs are 20-23 nucleotides long and are synthesized from double-stranded RNAs such as hairpins with a long, perfect stem or sense-antisense transcript hybrids. New members of endo-siRNA also include trans-acting siRNA(tasiRNAs), repeatassociated siRNAs, scan RNA (scnRNAs), long small interfering RNAs (IsiRNAs) [7,8]. Both miRNA and siRNA have been found in a single-stranded form with their effector complexes [3].

3.piRNA: The piRNAsaresingle-stranded RNAs, derivedwithout dicer cleavage from distinct piRNA clusters. These were discovered as 25-33 nucleotides small RNAs, transcribed from repetitive elements of transposons. They stabilize germline cells by repressing transposon elements particularly in spermatogenesis $[6,8,9]$. They are named as piRNAs because they bind PIWI [P element-induced wimpy testes] proteins to execute their jobs in the nucleus and cytoplasm [86].

Both miRNA and siRNAs bind to the members of the Ago clade of Argonaut proteins whereas piRNA bind to members of PIWI clade [3].

\section{What led to the serendipitous discovery of RNAi?} RNAi was first discovered in plants when enhancing the color of petunias via plasmid containing chalcone synthase gene led to co-silencing of its transgene and homologous genewhich resulted in totally white or patterned flowers with white or pale non-clonal sectors on a wild-type pigmented background [10]. During the same time, Dougherty's lab discovered the Tobacco Etch Virus (TEV) coat protein antisense RNA induced resistance against its infection in Nicotiana tabacum plants [11]. Another milestone in RNAi was made by Andrew Fire and Craig C. Mello in Caenorhabditis elegans, double-stranded RNA but not the single-stranded RNA against unc-22, unc54 , fem-1, and $h / h-1$ genes successfully silenced the targeted genes which led them to 2006Nobel Prize in Physiology or Medicine [12-14]. But all of this started from the discoveries of small temporal RNAs by Lee et al., 1993 [15] and Reinhart et al., 2000 [16]. The word RNA made a different meaning after Dr. Lee's discovery of the lin-4 gene that codes a precursor of 61 nucleotides to make $22 \mathrm{nt}$ miRNA. This $22 \mathrm{nt}$ miRNA represses lin-14 by complementarily binding at 7 sites of its $3^{\prime}$ UTR $[16,17]$. The expression of lin-14 is required to transit $C$. elegans from the first larval stage to the second larval stage. From lin-4 to let-7 in C. elegans, Drosophila, soon non-coding RNAs were discovered in humans, other bilateral animals, fungi, algae, plants and certain DNA viruses [10].

\section{How are small RNA molecules made?}

They are derived from larger RNA precursors, imperfect hairpin loop structure in case of miRNA, and perfect double-stranded RNA in case of siRNA. Both miRNA and siRNA genes at first are transcribed by RNA polymerase II and are known as primary miRNAs or pri-miRNAs or pri-siRNAs [18]. There are three major steps of biogenesis of mature and processed small RNA molecules (Figure 1).

1. The pri-miRNAs are processed into precursor miRNAs (pre-miRNAs) by the Microprocessor complex, consisting of the ribonuclease RNase III Drosha and DiGeorge Syndrome Critical Region 8 (DGCR8).

2. After synthesis of pre-miRNAs takes place in the nucleus they are exported into the cytoplasm by the Exportin 5 protein, where they are processed by a specialized RNase III-like enzyme named Dicer into smaller dsRNA molecules. Each pre-miRNA has two strands; the antisense strand to the targeted mRNA is referred to as the guide strand and its base-paired sense strand known as the passenger strand $[19,20]$.

3. Both pre-miRNA strands are transferred by Dicer and its cofactors TRBP (TAR RNA binding protein) and PACT (Protein kinase RNA activator) into an Argonaute (AGO2 in mammals) containing RISC complex.This complex formation first destroys the passenger strand and then only the guide strand in RISC-complex is targeted at the mRNA, which can degrade the target mRNA or destabilize it to block the translation [19].

Dicer is not always required for the processing of pre-miRNA. There are two ways of miRNA processing- canonical or nonconical. Canonical miRNAs processing depends upon the dicer and non-canonical miRNA does not require Dicer and Microprocessor complex. For example, the mirtron pathway, which is found in $\boldsymbol{D}$. melanogaster and $\boldsymbol{C}$. elegans, produces pre-miRNAs by the processing of introns by spliceosomes and debranching enzymes (not by dicer) in the nucleus, is an example ofthe non-canonical pathway [19]. The target mRNA recognition is initiated by a short nucleotide stretch at the $5^{\prime}$ end of the miRNA (position 2-8), the so-called seed sequence, accompanied by various degrees of base pairing at the $-3^{\prime}$ end [19]. In contrast to miRNA, siRNA target recognition requires base pairing of entire small RNA and subsequent target cleavage by AGO2. Guide pre-miRNA targets the RISC complex to $3^{\prime} U T R$ or other parts of mRNA results in translational inhibition, mRNA cleavage, de-adenylation, or histone and DNA methylation [3].

RNA interference as a vaccine

We do not live in 1,000 AD anymore where a charlatan would 


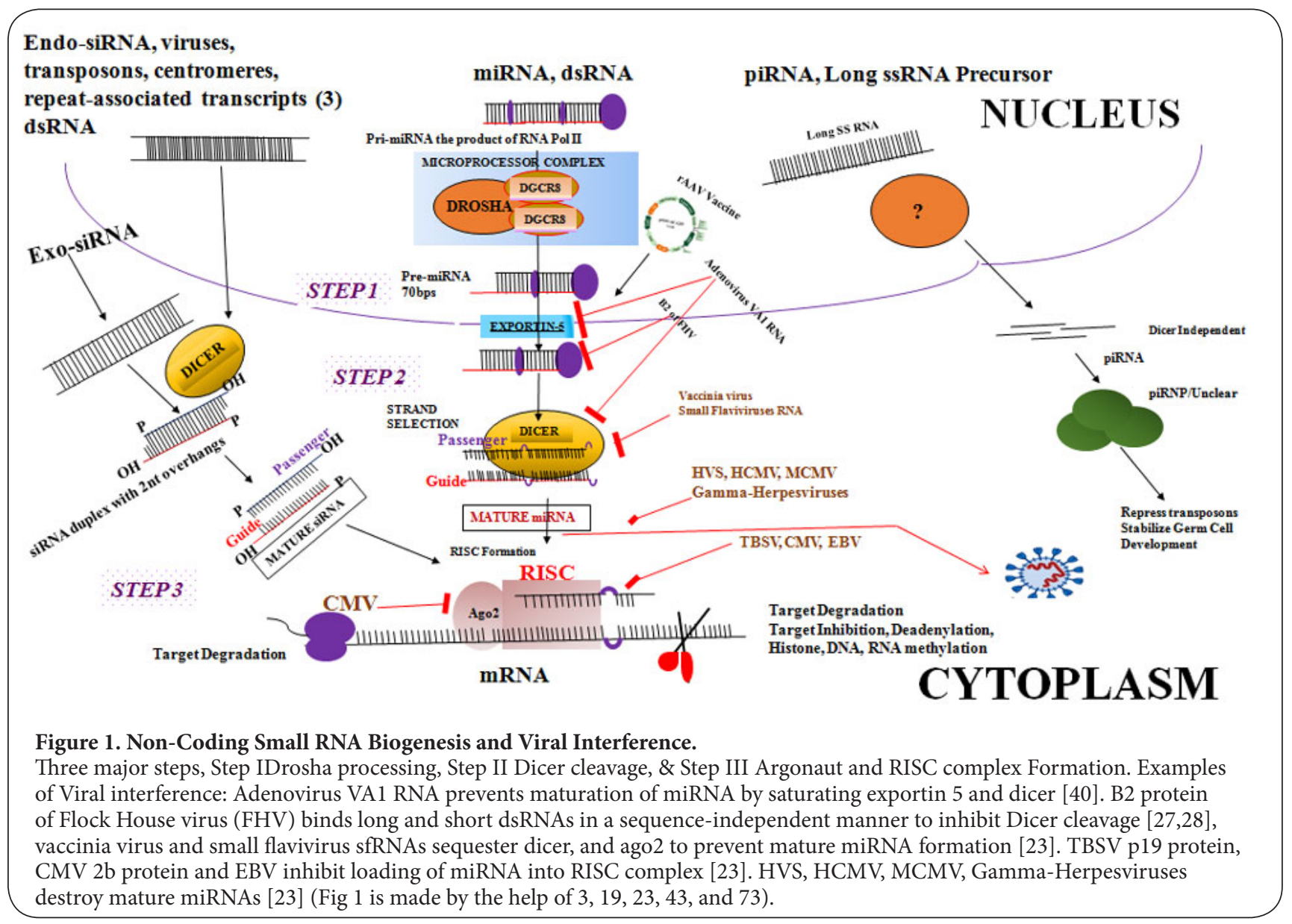

make a cut in the skin and transfers smallpox scabs from a patient into a normal human to immunize against smallpox. Our vast information on immunology and a spectrum of lab tools are at an edge in a way that we should be able to temporarily provide some kind of vaccine against any outbreak at any time.

The first experiment where RNAi was used as therapeutics was to prevent hepatitis. When mouse hepatocytes were injected with Fas siRNA they were resistant to Fas-induced apoptosis and hepatocyte necrosis upon exposing cells to Fas antibody or concanavalin-A (ConA). These experiments demonstrated the therapeutic value of Fas siRNA for preventing liver insults induced by viral, autoimmune hepatitis, and liver disorders [21,22]. Can we use small ncRNA molecules as a treatment against viral infections? But we first start with what happens in the waging battle between virus and host?

\section{Telltale of Virus vs Host RNAi}

How do cells distinguish between self and viral DS-, SS-DNA, or RNA and their modified forms?

Viruses contain different types of the genome like long dsDNA, dsRNA, 5'triphosphate, or 5'-diphosphate dsRNA, ssRNA, CpG motifs in ssDNA, and short dsDNA with guanosine-containing overhangs $[\mathbf{1 9}, \mathbf{2 3}, \mathbf{2 4}]$. The host cell can distinguish itself from nonself-genomic material. Cells have evolved a system of identifying own nucleic acid material from the foreign one by modifying it, for example, 2'Omethylation at the N1 position of capped RNA eliminates recognition by RIGI and IFIT1, but if 2'-Omethyl groups or other modifications such as pseudouridine are added at internal positions of RNA that prevents recognition by TLR7, TLR8, and MDA5 [24]. The C5 methylation of CpG motifs in DNA abolishes TLR9 recognition [24]. Vaccinia virus expresses an enzyme poly-A polymerase known as VP55, which is not only responsible for polyadenylation of viral mRNAs but also cellular miRNAs that results in degradation of mature miRNAs to allow viral sustenance, maybe that is how cellular RNAs and modified miRNAs were evolved for 2'-Omethylation to avoid degradation $[40,41,42,45]$.

The immune response to viral genomes depends upon their structure, concentration or availability, localizationinside, or outside the cell [24]. There are different types of cell receptors to recognize different nucleic acid molecules. These are categorized into first-line and second-line nucleic acid receptors. The pattern recognition receptors (PRRs) of the Toll-like receptor (TLR3, TLR7, TLR8, \& TLR9); the RIGllike receptor (RLR) family of RNA sensors (also known as DDX58); 
Aneja et al. Microbiology Discovery 2020,

melanoma differentiation-associated gene 5(MDA5; also known as IFIH1); the DNA sensors absent in melanoma 2 (AIM2) and cyclic GMP-AMP synthetase (cGAS) come under first-line receptors category $[19,23,24]$ (Figure 2). These firstline receptors instigate a cascade of signaling events that involve MYD88, TRIF, IRAKS, TRAFs, transcription factors like, nuclear factor-KB (NFKB) and IFN-regulatory factor 3 (IRF3), IRF7, and are dominated by type I interferon response and IFN-stimulatory genes (ISGs) [24] (Figure 2).

After Interferon is expressed; it is translocated across the cell membrane; it can signal in an autocrine or a paracrine way via the interferon-a/b receptor consisting of two subu- nits, IFNAR-1 (IFNAR; IFN receptor) and IFNAR2, this leads to phosphorylation of STAT transcription factors through the JAK-STAT pathway. STAT1 and STAT2 now heterodimerize and translocate to the nucleus to activate a broad range of ISGs (Figure 2). Second-line category receptors comprise nucleic acid receptors with direct antiviral activity, for example, doublestranded RNA (dsRNA)-activated protein kinase R (PKR; also known as elF2AK2), 2'5'oligoadenylate synthetase 1 (OAS1), adenosine deaminase acting on RNA 1 (ADAR1) [24], PKR, OAS1, and ADAE1 are also known as ISGs. The major difference between first-line and second-line nucleic acid receptors is that the second line receptors do not induce an immune

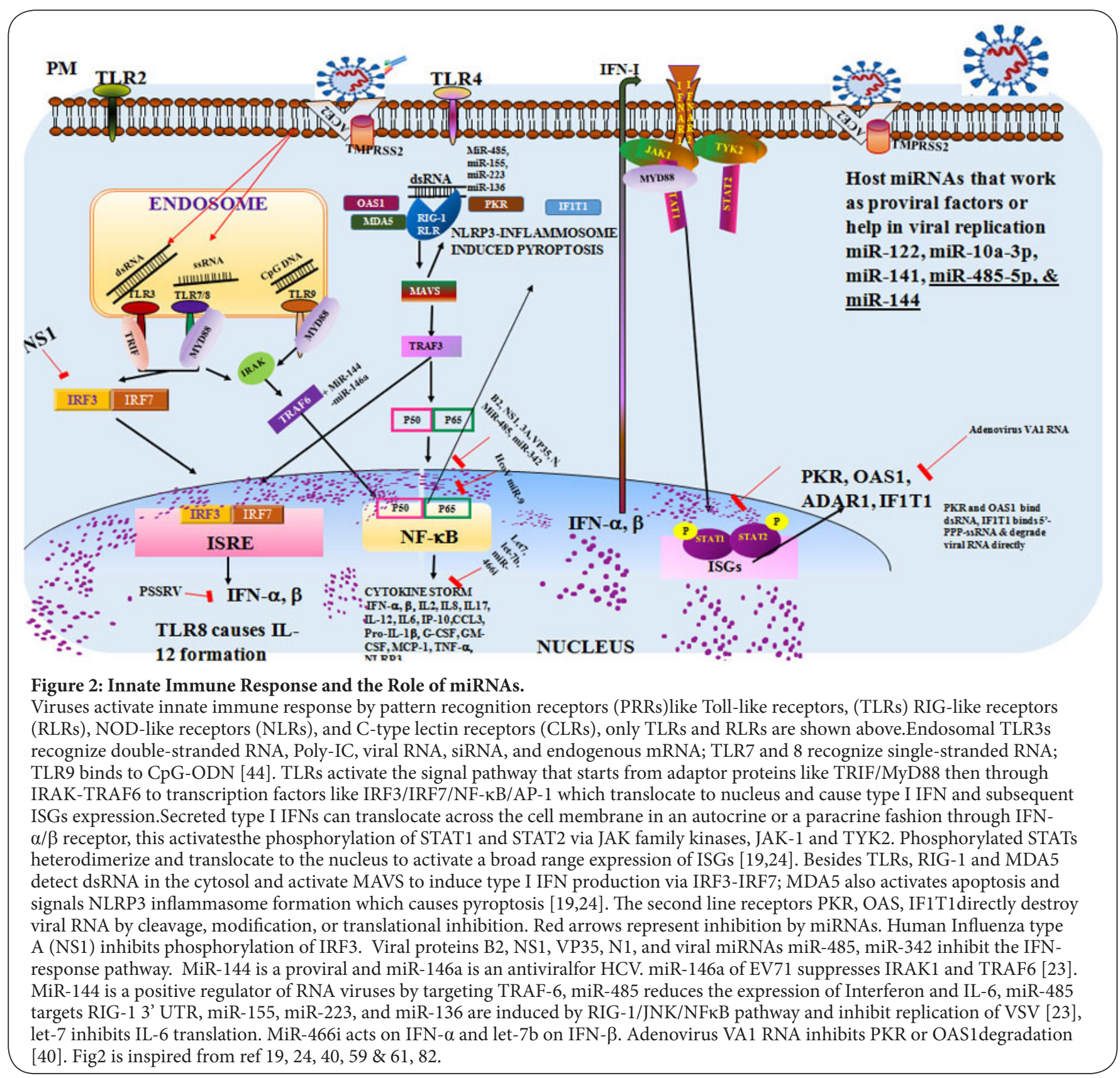


response by transcription factors or cytokines but directly destroy viral RNA by cleavage, modification, or translational inhibition [24]. Different TLRs responses lead to different outcomes, for example, TLR7 and TLR9 on plasmacytoid dendritic cells lead to type I IFN production while TLR8 on myeloid cells release IL-12 [24] (Figure 2). Overall excitement of innate immune response causes a storm of cytokines, chemokines, antiviral proteins that directly or indirectly, with or without adaptive immune response, cause the end of the viral realm by preventing viral replication, transcription, translation, virus assembly which ultimately leads to cell death including apoptosis, necroptosis, and pyroptosis.

\section{Raging battle of proviral versus antiviral factors}

From first-line to second-line nucleic acid receptors as mentioned above, then to IRF3/IRF7, STAT1 or STAT2, NF-kB, signaling transcription factors cascade to finally type I and type III interferon response, and expression of interferon stimulatory genes, anywhere in these steps host and virus deploy an arm of miRNAs on this raging war. The host makes miRNAs to destroy viruses and viruses make viral own miRNAs, and virulence factors (VSRs) to degrade host miRNAs and deploy host proteins for its multiplication [23]. On the other hand, host miRNAs destroy viral proviral factors which are important for viral replication. The virus is extremely clever, it can also use its genomic RNA as a sponge or sequester of endogenous host miRNA molecules, to help in its replication, these RNA sequences that share binding sites with host miRNA are known as competitive viral and host RNAs (cvhRNA) [25]. Host miRNA can also act as proviral factors and inhibit antiviral host factors, for example, miR-122, that would facilitate viral replication. Here are a few more examples and also see Table 1.

Table1. Examples of Host and Viral miRNAs involved in viral infections.

\begin{tabular}{|c|c|c|}
\hline Type of Virus & MiRNA (Host-made) & Viral Replication \\
\hline Hepatitis C (HCV) & $\begin{array}{l}\text { miR-196, miR-296, miR-351, } \\
\text { miR-431, miR-448 }\end{array}$ & Inhibits \\
\hline Vesicular stomatitis (VSV) & miR-155 \& miR-223 & Inhibits \\
\hline Epstein-Barr (EBV) & miR-155 & Inhibits \\
\hline Dengue virus2 (DENV2) & miR-150 & Inhibits \\
\hline Enterovirus 71 (EV71) & miR-296-5p, miR-23b & Inhibits \\
\hline Coxsackievirus B3 (CVB3) & $\operatorname{miR}-342-5 p$ & Inhibits \\
\hline Herpes simplex type 1 (HSV-1) & miR-138 & Inhibits \\
\hline DENV & miR-548g-3p & Inhibits \\
\hline Hepatitis C $(\mathrm{HCV})$ & $\operatorname{miR}-122$ & Helps \\
\hline Bovine viral diarrhea virus (BVDV) & miR-17, Let-7 & Helps \\
\hline Eastern equine encephalitis virus (EEEV) & miR-142-3p & Helps/Inhibits \\
\hline \multicolumn{3}{|c|}{ 2.Host miRNAs can degrade host proviral factors that help viral replication } \\
\hline Virus & MiRNA & Host Protein \\
\hline DENV2 & miR-223 & $\begin{array}{l}\text { downregulates the microtubule destabilizing } \\
\text { protein stathmin } 1 \text { (STMN1) }\end{array}$ \\
\hline Herpesviruses and alphaviruses & miR-199a-3p & $\begin{array}{l}\text { downregulation of ERK/MAPK, oxidative } \\
\text { stress and PI3K/AKT pathway }\end{array}$ \\
\hline WNV & miR-532-5p & $\begin{array}{l}\text { downregulates SESTD1 } \\
\text { (SEC14 and spectrin domains 1) and TAB3 } \\
\text { (TGF-beta activated } \\
\text { kinase 1/MAP3K7 binding protein } 3 \text { ) } \\
\text { mRNAs }\end{array}$ \\
\hline \multicolumn{3}{|l|}{ 3.Viral miRNAs } \\
\hline Virus & Non-coding RNA & Function \\
\hline$\gamma$-herpesvirus saimiri (HVS) & miR-HSURs & Degrades miR-27 \\
\hline Human cytomegalovirus (HCMV)), & miRNA decay element (miRDE) & degrades miR-17 \\
\hline Epstein-Barr virus (EBV) & (BARTs) & Cancer development \\
\hline Herpes simplex virus 1 & miR-H2-3p & Viral latency \\
\hline West nile virus Kunjin (WNVkun) & Kun-miR-1 & Viral replication enhancement \\
\hline HIV-1 & HIV1-miR-H1 & $\begin{array}{l}\text { Cellular apoptosis induction } \\
\text { Viral protein } \mathrm{R}(\mathrm{Vpr}) \text { stabilization }\end{array}$ \\
\hline
\end{tabular}

From Bruscella et al., 2017 and Girardi et al., 2018. 
Aneja et al. Microbiology Discovery 2020,

Many viral dsRNA-binding proteins bind viral dsRNA to hide it from dsRNA sensors of the host, so that the first step of invasion is successful, for example, B2 protein of Nodamura virus, NS1, the nonstructural protein of influenza $A$ virus, the $3 A$ protein of human enterovirus 71 (HEV71), VP35 of Ebola virus, SARS coronavirus $\mathrm{N}$ protein, and yellow fever virus capsid protein, these dsRNA binding proteins are both viral suppressors of RNAi and interferon response pathway [19] (Figure 2). Human Influenza type A nonstructural protein (NS1) inhibits phosphorylation of IRF3 and its nuclear translocation to prevent transcription of interferon stimulatory genes $[23,26]$. The B2 protein of Flock House virus (FHV) binds long and short dsRNAs in a sequence-independent manner to inhibit Dicer cleavage (Figure 1) $[27,28]$. These examples are evidence that viral proteins hide their genome from host recognition; viral proteins inhibit both IFN response pathway and RNAi $[19,23]$.

When a virus enters the host cell, along with the signal cascade to the interferon response, transcription of antiviral siRNA and miRNAs, and suppressors of host RNAi armamentarium are all in high alert. But viruses can use these antivirals as proviral factors; many viral RNAs contain common binding sites of miRNAs with the host RNA. For example, liver-specific miR-122 helps stabilize, replication, and transcription of hepatitis $\mathrm{C}$ virus (HCV) RNA; miR-122 binds at 5'-UTR of HCV RNA genome stimulates viral translation, protects the genome from XRN-1 mediated degradation; miR-122 also competes with cellular poly(rC)-binding protein 2 (PCBP2) binding to the HCV RNA genome and thereby promotes replication and packaging of HCV [23,29-32] (Figure 2, Table 1). The miRNA profiling of $\mathrm{HCV}$ by NanoString $\mathrm{nCounter}$ miRNA expression assays in Huh7.5.1 cells and primary human hepatocytes found major miRNA candidates out of which three proviral and 9 antiviral miRNAs showed interaction with HCV RNA, the top hits miR-25, miR130-a/b, let-7a were downregulated by the virus [33,34]. Bovine viral diarrhea virus (BVDV) of pestiviruses hijacks miR-17 and let-7 for their genome stability and replication [35], miR-10a star strand (miR-10a-3p) directly targets CVB3 3D-coding sequence to help its replication [36]. Host miRNAs like miR-141 in enterovirus (EV)71-infected cells [37], miR-485-5p in Newcastle disease virus (NDV) and the H5N1 strain of influenza virus [38], miR-144 in influenza-A virus (IAV) [39] were proviral factors or helped in their viral replication by competing with important host proteins for translation or immune response. MiR-144 is a positive regulator of RNA viruses; it attenuates IRF-7 mediated immune response by targeting TRAF-6 (Figure 2). MiR-485 results in the reduction of IFN and IL6 during infection by NDV or IAV [40] (Figure 2) and helps in viral replication. Herpes simplex virus (HSV-1) miR-H11 can be degraded by vaccinia virus protein VP55 results in lower viral DNA synthesis, restriction of viral spreading, and low viral yields, the mechanism is still unclear [42]. MiR-199a-3p against herpesvirus (a, b, g), miR-34, miR-15, \& miR-517 against flaviviruses, suggests some strong antiviral miRNA therapeutic candidates [40].
Can RNAi be used as a diagnostic and therapeutic? What do we need in our quiver to be prepared for an outbreak in the future?

The above examples are evidence of fierce competition between virus and host. Can we learn and use some of these tricks against viral infections? Can we use host or viral miRNA or siRNA or antisense RNAs as an attenuated vaccine? Can we use RNAi therapeutics as intervention drugs against viral infections? Can RNAi be used as a gene therapy against viral infections?

The global antisense \& RNAi therapeutics market size is expected to reach USD 1.81 billion by 2025 [47], right now it is about 1.2 billion [47]. RNAi with miRNAs and siRNAs have been successfully used in both diagnostics as signature biomarkers for a particular disease, foreign infection, and the progression of the disease and as a prognostic marker [47-53]. The clinical studies database clinicaltrials.gov includes phase 4 trials with selective miRNAs as biomarkers. These trials have assessed or are actively recruiting patients to examine the profiles of these ncRNA transcripts in a range of health conditions such as diabetes, coronary heart disease, breast cancer, lupus, epilepsy, depressive disorder, stroke, Addison's disease, influenza, liver disease, and even toxic exposure to agents such as acetaminophen [48]. There is a great need and competition in RNAi as clinical intervention drugs. In 2018, the FDA approved the siRNA drug, Patisiran, for a rare polyneuropathy caused by hereditary transthyretin-mediated (hATTR) amyloidosis. It works by binding and degrading the messenger RNA transcript for transthyretin $[\mathbf{5 4 , 5 5 ]}$. Many miRNA drugs like MRG110 against miR-92 antiangiogenic miRNA that prevent wound repair in diabetics [56]; MRG-201 for miR-29 to treat keloid and scar tissue formations; MRG-106 for miR-155 in T-cell lymphoma patients are under phase 1 or phase 2 trials [48]. Regulus has announced new miRNA drug RGLS5579 in 2019, this miRNA targets miR-10b for glioblastoma multiforme treatment [48]. First siRNA treatment in human started with ALN-RSV01, a 19 bp RNA duplex with two [2'-deoxy] thymidine overhangs on both 3 ' ends to prevent its nuclease degradation, a single site siRNA targeted to the nucleocapsid gene of RSV. The ALN-RSV01 relieves bronchiolitis obliterans when administered by intranasal spray. This drug is made by Alnylam Pharmaceuticals and has reduced a $38 \%$ decrease in the number of infected people $[62,63]$. At least eight anti-HBV siRNAs are in clinical trials [62]. The first synthetic miR-34 was developed in 2013 for the treatment of hepatocellular carcinoma [63]. There has been a success in generating synthetic miRNAs, packing in liposomes, and transfected into the mononuclear cells of peripheral blood. These protocols enhance TNF- a that favors the innate immune response. The PR8-amiR-93NP virus was generated by inserting an expression cassette for miR-93 between viral genes encoding non-structural proteins in an attenuated Influenza virus (IV), and this miRNA specifically targets the nucleoproteins of the IV. This vaccine, administered intranasally, conferred 
immunity against several heterologous viral strains $[63,70]$. New attenuated vaccines are in trials containing attenuated viruses that are loaded with an expression cassette encoding a synthetic designed miRNA that targets the structural protein of the virus.

\section{How about RNAi against COVID-19, Status Quo}

Coronaviruses are enveloped, non-segmented viruses that contain positive-sense single-stranded RNA; the viral genome is in the size from 26 to 32 kilobases, the largest known viral RNA genome [57]. Most of the cells containing cell membranebound angiotensin-converting enzyme 2 (ACE2) and associated proteases, transmembrane protease serine 2 (TMPRSS2), and Cathepsin $L(C T S L)$, are identified as the mediators of SARS CoV2 cellular entry $[58,59]$. Single-cell RNA sequencing datasets (sc-RNA-seq) from healthy donors by the Human Cell Atlas consortium and multiple published and unpublished datasets in multiple human tissues showed that ACEll expression was low in all datasets but was expressed in cells from multiple tissues, like, airways, cornea, esophagus, ileum, colon, liver, gallbladder, heart, kidney and testis. TMPRSS2 had a broader distribution. Both ACE2 and TMPRSS2 were co-expressed in respiratory tree, cornea, esophagus, ileum, colon, gallbladder and common bile duct cells $[58,59]$, but ACE2 and TMPRSS2 were highly expressed in a nasal goblet and ciliated cells. These cells are the locus and reservoir of viral outbreak and transmission through nasal infectious droplets and perhaps intranasal spray or vaccine can be effective in stymying its spread. These datasets expression studies also suggest that once SARS-CoV-2 has entered the respiratory tract, it can attack any cell because most of the cells contain ACE2 or TMPRSS2 and some cellscontain both. Upon looking at the genes coexpressed with ACE2 across all cells within the lung epithelial cell dataset, the interesting finding is that genes that perform innate and antiviral immune functions like IDO1, IRAK3, NOS2, TNFSF10, OAS1, and MX1 are co-expressed with ACE2 [58].

Inflammatory cytokine storm is the hallmark of COVID-19 which leads to plasma leakage, vascular permeability, and disseminated vascular coagulation and uncontrolled inflammatory response that account for life-threatening respiratory symptoms [60]. The plasma concentrations of IL-1 $\beta$, IL-1 ra, IL-7, IL-8, IL-9, IL-10, basic FGF, G-CSF, GM-CSF, IFN- - , IP-10, MCP-1, MIP-1 $\alpha$, MIP-1 $\beta$, PDGF, TNFa, and VEGF were higher in both ICU (intensive care unit) patients and non-ICU patients than in healthy adults. Moreover, when comparing ICU and nonICU patients, plasma concentrations of IL-2, IL-7, IL-10, G-CSF, IP-10, MCP-1, MIP-1a, and TNFa were higher in ICU patients than non-ICU patients, evidence that the cytokine storm is correlated with disease severity [61] (Figure 2). Selected drugs like tocilizumab-sarilumab, baricitinib-fedratinib, and fingolimod are in consideration to manage this storm [60].

Academic laboratories, biotech companies, and the pharmaceutical industries are all involved in the clinical research efforts against COVID-19. There is no cure for COVID-19 ex- cept for a few therapeutic drugs that are available for other viruses, e.g., chloroquine and hydroxychloroquine, anti-viral remdesivir, Kaletra which is a combination of lopinavir and ritonavir that works against HIV, favipiravir, and arbidol [65]. In one of the tissue culture experiments for testing efficacy of remdesivir, after 23 passages in Calu-3 2B4 cells, SARS CoV viral RNA-dependent RNA polymerase enzyme mutations at F480L and V557L showed resistance to remdesivir even though these mutations did not affect the pathogenicity of remdesivir in mice [66]. TMPRSS2 cleaves and activates SARSCoV-2 spike protein and helps in the viral entrance. Camostat mesylate and $\mathrm{K} 11777$, a cysteine protease inhibitor inhibits the enzymatic activity of TMPRSS2 [65] (Figure 2).

Monoclonal antibodies represent a major class of passiveimmunotherapy treatment against viral infections. Convalescent plasma has been successfully used in various infections like SARS, MERs, influenza, and was started in the Spanish flu pandemic [67]. The different convalescent blood products to achieve artificial acquired passive immunity have been categorized into 4 types (i) convalescent whole blood (CWB), convalescent plasma (CP) or convalescent serum (CS); [ii] pooled human immunoglobulin (Ig) for intravenous or intramuscular administration; [iii] high-titer human Ig; and (iv) polyclonal or monoclonal antibodies [67]. Antibodies from a patient recovering from a viral infection can be transferred or can be manufactured in the lab. The specific monoclonal antibody raised against the receptor-binding domain in spike (S) protein can stop the viral entry. A pair of antibodies isolated from the patient's convalescent plasma$\mathrm{B} 38$ and $\mathrm{H} 4$ block the binding between virus S-protein RBD and cellular receptor ACE2 [68]. The human 47D11 derived from SARS and SARS-CoV-2 and reformatted to a fully human immunoglobulin is the first monoclonal against spike RBD that cross-neutralizes SARS and SARS-CoV-2 in a mechanism independent of receptor-binding inhibition [69]. Companies that are in process of COVID-19 vaccine are Moderna, Inovio, the University of Oxford in England, the University of Queensland in Australia, J\& J, Sanofi, etc.

\section{Are there any RNAi therapies for COVID-19?}

There are three major steps of COVID-19 infection- viral entry, viral replication, viral maturation, and release. We can design small non-coding RNA molecules to halt this virus at any of these steps. We already have drugs, vaccines, antibodies, as patent or in clinical trials for all of these steps. How about RNAi? Cells affected by human coronavirus activate signal cascades that increase NF-KB1 mRNA and miR-9 expression, miR-9 reduces the translation of NF-KB and can be competed away by OC43 and which allows NFkB translation [63], so miR-9 isa candidate or RNAi since it is induced by the coronavirus to inhibit NFkB pathway. SARS-HcoV mainly infectsbronchioalveolar stem cells (BASCs) where they induce overexpression of miR-574-5p and miR-214.Some proteins of the SARS-HcoV viral nucleocapsid downregulate miR-224 and miR-98 expression 
Aneja et al. Microbiology Discovery 2020,

http://www.hoajonline.com/journals/pdf/2052-6180-8-1.pdf

doi: 10.7243/2052-6180-8-1

in BASCsand thatcontrol several stages of their differentiation as well as pro-inflammatory cytokine production $[63,64]$. So we know miR-9, miR-574-5p, miR-214, miR-224, and miR-98 are important targets of coronaviruses and can be used as RNAi therapeutics.

A total of 188 patents are directly associated with antiSARS and anti-MERS vaccines with a demonstrated immune response [71] (Table 2). Based upon clinicltrials.govout of 127 potential COVID-19 vaccinesten are in phase III trial. SARSCoV-2 mRNA-1273 vaccine which is in phase trial Illhas shown a safe and strong response in older adults. This vaccine is codeveloped by NIAID and Moderna (87). This vaccine contains mRNA-1273 that codes for two prolines substituted at the top of the central helix full length SARS-CoV-2 spike glycoprotein trimer, S-2P. The mRNA is encapsulated in lipid nanoparticles. Other few examples of COVID-19 vaccines are given in Table 2.

There are anti-siRNA patents against four major structural proteins, spike (S) protein, membrane (M) protein, nucleocapsid (N) protein, and envelope (E) proteins of SARS virus, some of them are more than $70 \%$ effective. One of the patent applications, CN1569233 discloses siRNAs that target SARS genes encoding RNA-dependent RNA polymerase, helicase, $\mathrm{N}$ protein, and proteolytic enzymes (Table 2). These siRNAs were able to inhibit or kill $50-90 \%$ of the SARS virus BJ01 strain, with the proteolytic enzyme-targeting siRNAs being the most effective [71]. RNA aptamers, ribozymes, and antisense oligonucleotides have been developed to fight against SARS by reducing its severity, preventing its multiplication steps, detecting, or diagnosing it accurately.

Since human adeno-associated virus (AAV) is a frequent cause of upper respiratory infections, AAV isdesigned to deliver genes, miRNAs, and siRNAs in the lungs. AAV is about $4.7 \mathrm{~kb}$ and has a positive or negative-sensed single-stranded DNA. Because of AAV's low immune response, it can target both dividing and quiescent cells, makesAAV an attractive vector for gene delivery. Recombinant AAV (rAAV) is constructed by replacing the viral structural(cap) and packaging (rep) genes with the desired transgene along with promoter and polyadenylation sequences $[72,73]$ (Figure 1).

SARS-CoV-2s 3' region of about $10,000 \mathrm{~kb}$ encodes for the $S, E, M$, and $N$ functional proteins, specific miRNAs or siRNAs can be designed to target the sequences for degradation by RISC complex [73]. It can be designed as a vaccine or an intervention drug. There is another great scientific adventure on the horizon, synthesis of asymmetrical siRNAs to target the $5^{\prime}$ and $3^{\prime}$ UTRs and 10 viral ORFs of the $\sim 29 \mathrm{~kb}$ SARS-CoV-2

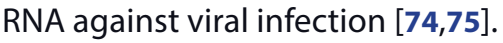

\section{But there is another twist to this story}

Our diet has many functions including, sustenance, immunomodulatory [81], neuro-modulatory, cardiovascular regulation, auto-immune disorders, and viral infections [79]. What we eat controls our health, personality, and our chance of survival; our genes, miRNAs, transposon elements, proteins, etc., work in synchrony to determine our surrender or victory over known human medical conditions and infections. Since the discovery of rice miR-168a by Zhang et al., 2012, plant-microRNAs have been a hot topic of discussion. The rice miR-168a targets mammalian LDLRAP1 which increases LDL levels in plasma [76]. This has set out scientists all over the world to investigate diet plant miRNAs for the cure and prevention of human medical conditions. How do plant-based diet and plant microRNAs contribute to human well-being?

Plants produce miRNAs that regulate virus replication. MiR2911 from Lonicera japonica, and Lonicera periclymenumtarget and inhibit Influenza A virus (H1N1, H5N1, H7N9) replication in mice $[77,78]$. The miR159 present in plants including, Arabidopsis thaliana, Glycine max, and Brassica oleracea, has a role in breast cancer suppression by targeting the TCF-7 gene [79]. MiR-156a from green vegetables acts as a vasoprotective molecule by targeting the junction adhesive molecule-A (JAM-A), it reduces cytokine-induced monocytes adhesion in endothelial cells [79]. American sweetgum green fruits are used for making an antiflu tincture. The key ingredient in Tamiflu ${ }^{\oplus}$ is oseltamivir phosphate, of which shikimic acid is a precursor, and it is present in green seeds of American sweetgum [80]. We need to do more experiments on deciduous trees of the eastern seaboard to investigate their medicinal importance.

\section{Future Prospective}

We are still far from fully understanding the molecular mechanisms of networking pathways run by non-coding RNAs in different cells, cell types, plants, and animals, and how they benefit or destroy foreign invaders. There are already many antimiRNA or antisiRNA patents and clinical trials against a range of health conditions such as diabetes, coronary heart disease, breast cancer, lupus, epilepsy, depressive disorder, stroke, Addison's disease, influenza, liver disease, and even toxic exposure to agents such as acetaminophen [48]. We already have patents and clinical trials against Influenza, HBV, HCV, RSV, HIV, and SARS-CoV and SARS-CoV-2. For example, ALN-RSV01, against RSV, administered by intranasal spray, is made by Alnylam pharmaceuticals, has reduced $38 \%$ infected patients $[62,63]$. Alnylam also has 350 siRNAs in a trial against SARS-CoV-2; Sirnaomica has siRNA formulation via nebulizer Table 2 [85]. The PR8-amiR-93NP virus particle is designed against the Influenza virus and its heterologous strains $[73,70]$. RG101 is N-acetylgalactosamine conjugated antisense oligonucleotide which improves its delivery in hepatocytes for sequestration of miR-122 to reduce the HBV burden and relief from its symptoms in patients [40]. These examples are evidence of tremendous hard work by the scientific workforce in the direction of improving healthcare using RNAi and we hope for the SARS-CoV-2 also.

Small non-coding RNAs like miRNA, siRNA, and anti-sense RNAs are very interesting molecules because they induce low immunogenicity, and show cross-species conservation and can be tested in different animal models. But there are 
Aneja et al. Microbiology Discovery 2020,

http://www.hoajonline.com/journals/pdf/2052-6180-8-1.pdf

Table 2. Drugs, RNA therapies, and other treatments for SARS-CoV-2.

\begin{tabular}{|c|c|c|}
\hline Group & Mechanism of Action & Examples \\
\hline Viral entry inhibitors & $\begin{array}{l}\text { Increase in endosomal } \mathrm{pH} \text { that inhibits viral- } \\
\text { cell fusion, proper genome release and efficient } \\
\text { infection; Interferes with glycosylation of } \\
\text { ACE2 }\end{array}$ & Hydroxychloroquine, Chloroquine \\
\hline Viral entry inhibitors & $\begin{array}{l}\text { an inhibitor that may disrupt the binding of } \\
\text { viral envelope protein to host cells and prevent } \\
\text { viral entry to the target cell, inhibits intracel- } \\
\text { lular vesicular trafficking }\end{array}$ & Arbidol \\
\hline \multirow[t]{2}{*}{$\begin{array}{l}\text { Inhibitors of viral RNA polymerase /RNA } \\
\text { synthesis }\end{array}$} & $\begin{array}{l}\text { Prodrug of Adenosine nucleotide analogue, } \\
\text { inhibits viral RdRp }\end{array}$ & Remdesivir \\
\hline & $\begin{array}{l}\text { Guanosine nucleotide analogue, prodrug, } \\
\text { inhibits viral RdRp }\end{array}$ & Favipiravir \\
\hline Inhibitors of viral protein synthesis & $\begin{array}{l}\text { Protease inhibitor that may inhibit 3CLpro or } \\
\text { PLpro }\end{array}$ & Lopinavir/ritonavir aka Kaletra \\
\hline Viral Protease & $\begin{array}{l}\text { protease inhibitors that may inhibit the viral } \\
\text { proteases: 3CLpro or PLpro }\end{array}$ & \\
\hline JAK signaling inhibitors & $\begin{array}{l}\text { targeting IL-6, inhibiting IL6/JAK/STAT } \\
\text { signaling, attenuate the host inflammatory } \\
\text { response associated with massive pro- } \\
\text { inflammatory cytokine and chemokine storm }\end{array}$ & Baricitinib-Fedratinib, Fingolimod \\
\hline \multicolumn{3}{|l|}{ RNA Therapies } \\
\hline RNA Therapies & $\begin{array}{l}\text { Anti-miRNA, anti-siRNA, ribozymes, RNA } \\
\text { aptamer, anti-sense oligos, recombinant AAV }\end{array}$ & \\
\hline \multirow[t]{6}{*}{ Patents } & US20040192626, siRNA, microRNA, shRNA & Various regions of SARS \& MERS \\
\hline & WO2004092383 & Various regions of SARS \& MERS \\
\hline & $\begin{array}{l}\text { WO2018227440 MicroRNA inhibitor } \\
\text { microRNA200c-3p }\end{array}$ & $\begin{array}{l}\text { Applications of mir-200c-3p microR- } \\
\text { NA in treating lung injuries }\end{array}$ \\
\hline & CN101173275 & $\begin{array}{l}\text { siRNAs targeting coronavirus proteins } \\
\mathrm{M}, \mathrm{N} \text {, or E of SARS }\end{array}$ \\
\hline & US20050004063 & $\begin{array}{l}\text { siRNAs targeting replicase and RdRp } \\
\text { region of SARS }\end{array}$ \\
\hline & CN1569233 & $\begin{array}{l}\text { siRNAs targeting RdRp, helicase, } \\
\text { nucleoprotein N, and proteolytic } \\
\text { enzymes of SARS }\end{array}$ \\
\hline \multicolumn{3}{|l|}{ Companies } \\
\hline Alnylam Pharmaceuticals & 350 siRNAs in trial & Against SARS-CoV-2 \\
\hline Sirnaomica & siRNA formulation by a nebulizer & Against SARS-CoV-2 \\
\hline \multicolumn{3}{|l|}{ Other treatments } \\
\hline mRNA-1273 Vaccine & $\begin{array}{l}\text { prolines substitutedSARS-CoV-2 spike } \\
\text { glycoprotein trimer, S-2P (87) }\end{array}$ & Against SARS-CoV-2 \\
\hline BNT162B2 by Pfizer and Biontech & $\begin{array}{l}\text { A nucleoside-modified mRNA expresses spike } \\
\text { glycoprotein }(88)\end{array}$ & Against SARS-CoV-2 \\
\hline NVXCoV2373 & spike (S) protein\&saponin-based adjuvant (89) & Against SARS-CoV-2 \\
\hline JNJ-78436725 or Ad.26.COV2.S & $\begin{array}{l}\text { adenovirus serotype } 26 \text { (Ad26) expressing the } \\
\text { spike (S) (90) }\end{array}$ & Against SARS-CoV-2 \\
\hline MRT5500 by Sanofi & Spike glycoprotein S (91) & Against SARS-CoV-2 \\
\hline Convalescent Plasma & $\begin{array}{l}\text { Convalescent blood products, pooled human } \\
\text { immunoglobulins (IgG), mono or polyclonal } \\
\text { antibodies }\end{array}$ & $\begin{array}{l}\text { B38 and H4 (68) } \\
\text { Human 47D11(69) }\end{array}$ \\
\hline
\end{tabular}

RdRp, RNA-dependent RNA polymerase; coronavirus main protease, (3CLpro), and papain-like protease (PLpro), Table2 adapted from refs $60,65,68,69,71,83,84,85,87,88,89,90$, \& 91.

challenges with design, delivery of live attenuated vaccines, miRNA drugs, and off-target effects. Their safety and toxicity remain a controversial issue.
We are writing so much about different miRNA as a pro- or anti-viral, but all of these mechanisms will vary from lab to lab, the results could be contradictory, but perhaps we can find 
Aneja et al. Microbiology Discovery 2020,

http://www.hoajonline.com/journals/pdf/2052-6180-8-1.pdf

doi: $10.7243 / 2052-6180-8-1$

some commonalities in data that can lead us to therapeutical interventions and vaccines against COVID-19 and other viral infections. There is a big scope of plant miRNA therapeutics as a dietary supplement and future medicine.

\section{List of abbreviations \\ RNAi: RNA interference \\ HVS: Herpesvirus saimiri \\ HCMV: Human cytomegalovirus \\ MCMV: Murine cytomegalovirus \\ TBSV: Tomato bushy stunt virus \\ EBV: Epstein-barr virus \\ CMV: Cucumber mosaic virus \\ CVB3: Coxsackievirus B3 \\ FHV: Flock House virus}

VSV-G: Vesicular somatitis virus $G$ protein

IV: Influenza virus

HcoV: Human coronavirus

RSV: Respiratory syncytial virus

HBV: Hepatitis B virus

DGCR8: DiGeorge Sydrome Critical Region 8

RIG-1: Retinoic acid inducible gene I

5'ppp: 5'-triphosphate

IFN: Interferon

IFIT1: IFN-induced protein with tetratricopeptide repeats 1

IFNAR: IFN receptor

STAT: Signal transducer and activation of transcription

ISG; Interferon stimulatory genes

ADAR1: Adenosine deaminase acting on RNA 1

dsRNA: Double-stranded RNA

ssRNA: Single-stranded RNA

RBD: Receptor binding domain

IFIT1: IFN-induced protein with tetratricopeptide repeats 1

MDA5: Melanoma differentiation associated gene 5

NP: Nucleocapsid protein

OAS1: 2'-5'-oligoadenylate synthetase 1

PKR: Protein kinase $\mathrm{R}$

TLR: Toll-like receptor

TRAF6: Tumor necrosisfactor receptor-associated factor 6

TRAM: TRIF-related adaptermolecule

TRIF: TIR domain-containing adapter protein inducing IFN- $\beta$

MyD88: Myeloiddifferentiation primary response gene 88

MDA5: Melanoma differentiation-associatedgene 5

IRAK4: Interleukin 1 receptor-associated kinase 4

IRF: Interferon regulatory factor

NLRP3: NOD-, LRR- and pyrin domain-containing 3

\section{Competing interests}

The authors declare that they have no competing interests.

\section{Acknowledgements}

I am extremely thankful to Dr. AK for giving me inspiration to write this article. Thanks to Ms. Kinu Aneja for editing this article.

Authors' contributions
\begin{tabular}{|l|c|c|c|}
\hline Authors' contributions & KKA & ND & AK \\
\hline Research concept and design & $\checkmark$ & -- & -- \\
\hline Collection and/or assembly of data & $\checkmark$ & -- & -- \\
\hline Data analysis and interpretation & $\checkmark$ & $\checkmark$ & $\checkmark$ \\
\hline Writing the article & $\checkmark$ & -- & -- \\
\hline Critical revision of the article & $\checkmark$ & -- & $\checkmark$ \\
\hline Final approval of article & $\checkmark$ & -- & -- \\
\hline Statistical analysis & $\checkmark$ & -- & -- \\
\hline
\end{tabular}

\section{Publication history}

Editors: Antonio Pedro Fonseca, University of Porto, Portugal. Received: 26-Sept-2020 Revised: 20-Nov-2020

Accepted: 24-Nov-2020 Published: 17-Dec-2020

\section{References}

1. Mello, C., Conte, D. Revealing the world of RNA interference. Nature 431, 338-342 (2004).

2. Lyu, J. RNA silencing: Machinery in algae. Nature Plants 2, 16070 (2016).

3. Carthew, R. W., \& Sontheimer, E. J. (2009) Origins and Mechanisms of miRNAs and siRNAs. Cell, 136(4), 642-655. https://doi.org/10.1016/j. cell.2009.01.035

4. Bartel, D. (2004) MicroRNAs: Genomics, Biogenesis, Mechanism, and Function. Cell, Vol. 116, 281-297

5. Großhans, H., Filipowicz, W. The expanding world of small RNAs. Nature 451, 414-416 (2008). https://doi.org/10.1038/451414a

6. Piatek, M. J., \& Werner, A. (2014). Endogenous siRNAs: regulators of internal affairs. Biochemical Society transactions, 42(4), 1174-1179. https://doi.org/10.1042/BST20140068

7. Katiyar-Agarwal, S., Gao, S., Vivian-Smith, A., \& Jin, H. (2007). A novel class of bacteria-induced small RNAs in Arabidopsis. Genes \& development, 21(23), 3123-3134. https://doi.org/10.1101/gad.1595107

8. Ghildiyal, M., \& Zamore, P. D. (2009). Small silencing RNAs: an expanding universe. Nature reviews. Genetics, 10(2), 94-108. https://doi. org/10.1038/nrg2504

9. Siomi MC, Sato K, Pezic D, Aravin AA. PIWI-interacting small RNAs: the vanguard of genome defence. Nat Rev Mol Cell Biol. 2011; 12:246-258

10. Napoli C, Lemieux C, Jorgensen R (1990) Introduction of a chimeric chalcone synthase gene into Petunia results in reversible cosuppression of homologous genes in trans. Plant Cell 2:279-289

11. Lindbo J.A, Dougherty W. G. (1992) Untranslatable transcripts of the tobacco etch virus coat protein gene sequence can interfere with tobacco etch virus replication in transgenic plants and protoplasts. Virology. 1992;189(2):725-733. doi:10.1016/0042-6822(92)90595-g

12. Fire, A. Xu S, Montgomery MK, Kostas SA, Driver SE, Mello CC (1998). "Potent and specific genetic interference by double-stranded RNA in Caenorhabditis elegans". Nature. 391 (6669): 806-811

13. Daneholt B. "Advanced Information: RNA interference". The Nobel Prize in Physiology or Medicine 2006.

14. Ambros, V. MicroRNA pathways in flies and worms: growth, death, fat, stress, and timing. Cell 113, 673-676 (2003).

15. Lee, R.C., Feinbaum, R.L., and Ambros, V. (1993). Cell 75, 843-854.

16. Reinhart, B.J., Slack, F.J., Basson, M., Pasquinelli, A.E., Bettinger, J.C., Rougvie, A.E., Horvitz, H.R., and Ruvkun, G. (2000). The 21-nucleotide let-7RNA regulates developmental timing in Caenorhabditis elegans. Nature 403, (6772):901-906.

17. He, L., Hannon, G. MicroRNAs: small RNAs with a big role in gene regulation. Nat Rev Genet 5, 522-531 (2004). https://doi.org/10.1038/ nrg1379

18. Castel, Stephane E., and Robert A. Martienssen. "RNA interference in the nucleus: roles for small RNAs in transcription, epigenetics and beyond." Nature Reviews Genetics, vol. 14, no. 2, 2013, p. 100 
Aneja et al. Microbiology Discovery 2020,

19. Schuster, S., Miesen, P., \& van Rij, R. P. (2019). Antiviral RNAi in Insects and Mammals: Parallels and Differences. Viruses, 11(5), 448. https://doi. org/10.3390/v11050448

20. Pei, Y., Tuschl, T. On the art of identifying effective and specific siRNAs. Nat Methods 3, 670-676 (2006). https://doi.org/10.1038/ nmeth911

21. Song E, Lee SK, Wang J, et al. RNA interference targeting Fas protects mice from fulminant hepatitis. Nat Med. 2003;9(3):347-351.

22. Tan FL, Yin JQ. RNAi, a new therapeutic strategy against viral infection. Cell Res. 2004;14(6):460-466.

23. Bruscella, P., Bottini, S., Baudesson, C., Pawlotsky, J. M., Feray, C., \& Trabucchi, M. (2017). Viruses and miRNAs: More Friends than Foes. Frontiers in microbiology, 8, 824.

24. Schlee, Martin, and Gunther Hartmann. "Discriminating self from nonself in nucleic acid sensing." Nature Reviews Immunology, vol. 16, no. 9, 2016, p. 566

25. Li, C., Hu, J., Hao, J., Zhao, B., Wu, B., Sun, L., et al. (2014). Competitive virus and host RNAs: the interplay of a hidden virus and host interaction. Protein Cell 5, 348-356

26. Talon, J.; Horvath, C.M.; Polley, R.; Basler, C.F.; Muster, T.; Palese, P.; Garcia-Sastre, A. Activation of interferon regulatory factor 3 is inhibited by the influenza A virus NS1 protein. J. Virol. 2000, 74, 7989-7996

27. Luna, J. M., Scheel, T. K., Danino, T., Shaw, K. S., Mele, A., Fak, J. J., et al. (2015). Hepatitis C virus RNA functionally sequesters miR-122. Cell 160, 1099-1110. doi: 10.1016/j.cell.2015.02.025

28. Chao, J.A.; Lee, J.H.; Chapados, B.R.; Debler, E.W.; Schneemann, A.; Williamson, J.R. Dual modes of RNA-silencing suppression by flock house virus protein B2. Nat. Struct. Mol. Biol. 2005, 12, 952-957.

[CrossRef] [PubMed]

29. Lingel, A.; Simon, B.; Izaurralde, E.; Sattler, M. The structure of the flock house virus b2 protein, a viral suppressor of RNA interference, shows a novel mode of double-stranded RNA recognition. EMBO Rep. 2005, 6, 1149-1155. [CrossRef]

30. Shimakami, T., Yamane, D., Jangra, R. K., Kempf, B. J., Spaniel, C., Barton, D. J., et al. (2012). Stabilization of hepatitis $C$ virus RNA by an Ago2-miR-122 complex. Proc. Natl. Acad. Sci. U.S.A. 109, 941-946. doi: 10.1073/pnas.1112263109

31. Sedano, C. D., and Sarnow, P. (2014). Hepatitis C virus subverts liverspecific miR- 122 to protect the viral genome from exoribonuclease Xrn2. Cell Host Microbe 16, 257-264. doi: 10.1016/j.chom.2014.07.006

32. Masaki, T., Arend, K. C., Li, Y., Yamane, D.,McGivern, D. R., Kato, T., et al. (2015). miR-122 stimulates hepatitis C virus RNA synthesis by altering the balance of viral RNAs engaged in replication versus translation. Cell Host Microbe 17, 217-228.

33. Schult, P., Roth, H., Adams, R.L. et al. microRNA-122 amplifies hepatitis $C$ virus translation by shaping the structure of the internal ribosomal entry site. Nat Commun 9, 2613 (2018).

34. Li, Q., Lowey, B., Sodroski, C., Krishnamurthy, S., Alao, H., Cha, H., et al. (2017) Cellular microRNA networks regulate host dependency of hepatitis C virus infection. Nat. Commun. 8:1789. doi: 10.1038/s41467017-01954-x

35. Brochado-Kith, Ó., Gómez Sanz, A., Real, L. M., Crespo García, J., Ryan Murúa, P., Macías, J., Cabezas González, J., Troya, J., Pineda, J. A., Arias Loste, M. T., Díez Viñas, V., Jiménez-Sousa, M. Á., Medrano de Dios, L. M., Cuesta De la Plaza, I., Monzón Fernández, S., Resino García, S., \& Fernández-Rodríguez, A. (2019). MicroRNA Profile of HCV Spontaneous Clarified Individuals, Denotes Previous HCV Infection. Journal of clinical medicine, 8(6), 849. https://doi.org/10.3390/jcm8060849

36. Scheel, T. K., Luna, J. M., Liniger, M., Nishiuchi, E., Rozen-Gagnon, K., Shlomai, A., et al. (2016). A Broad RNA virus survey reveals both miRNA dependence and functional sequestration. Cell Host Microbe 19, 409-423. doi: 10.1016/j.chom.2016.02.007

37. Tong, L., Lin, L., Wu, S., Guo, Z., Wang, T., Qin, Y., et al. (2013). MiR-10a upregulates coxsackievirus $\mathrm{B} 3$ biosynthesis by targeting the 3D-coding sequence. Nucleic Acids Res. 41, 3760-3771. doi: 10.1093/nar/gkt058

38. Ho, B.-C., Yu, S.-L., Chen, J. J. W., Chang, S.-Y., Yan, B.-S., Hong, Q.-S., et al. (2011). Enterovirus-induced miR-141 contributes to shutoff of host protein translation by targeting the translation initiation factor elF4E. Cell HostMicrobe 9, 58-69. doi: 10.1016/j.chom.2010.12.001

39. Ingle, H., Kumar, S., Raut, A. A., Mishra, A., Kulkarni, D. D., Kameyama, T., et al. (2015). The microRNA miR-485 targets host and influenza virus transcripts to regulate antiviral immunity and restrict viral replication. Sci. Signal. 8:ra126. doi: 10.1126/scisignal.aab3183

40. Rosenberger, C. M., Podyminogin, R. L., Diercks, A. H., Treuting, P. M., Peschon, J. J., Rodriguez, D., et al. (2017). miR-144 attenuates the host response to influenza virus by targeting the TRAF6-IRF7 signaling axis. PLoS Pathog.13:e1006305. doi: 10.1371/journal.ppat.1006305

41. Girardi E, López P, Pfeffer S. On the Importance of Host MicroRNAs During Viral Infection. Front Genet. 2018;9:439. Published 2018 Oct 2. doi:10.3389/fgene.2018.00439

42. Backes, S., Shapiro, J. S., Sabin, L. R., Pham, A. M., Reyes, I., Moss, B., et al. (2012). Degradation of host micrornas by poxvirus Poly(A) polymerase reveals terminal RNA methylation as a protective antiviral mechanism. Cell Host Microbe 12, 200-210. doi: 10.1016/j.chom.2012.05.019

43. Zou, W., Zhou, X., Wang, L., Zhou, G. G., \& Chen, X. (2020). Degradation of Herpes Simplex Virus-1 Viral miRNA H11 by Vaccinia Virus Protein VP55 Attenuates Viral Replication. Frontiers in microbiology, 11, 717. https://doi.org/10.3389/fmicb.2020.00717

44. Sifuentes-Romero I, Milton SL, García-Gasca A. Post-transcriptional gene silencing by RNA interference in non-mammalian vertebrate systems: where do we stand? Mutat Res. 2011;728(3):158-171.

45. Krishnan J, Selvarajoo K, Tsuchiya M, Lee G, Choi S. Toll-like receptor signal transduction. Exp Mol Med. 2007;39(4):421-438. doi:10.1038/ emm.2007.47

46. Abbas YM, Pichlmair A, Górna MW, Superti-Furga G, Nagar B. Structural basis for viral 5'-PPP-RNA recognition by human IFIT proteins. Nature. 2013;494(7435):60-64. doi:10.1038/nature11783

47. Sanchita, Ritu Trivedi, Mehar Hasan Asif \& Prabodh Kumar Trivedi (2018) Dietary plant miRNAs as an augmented therapy: cross-kingdom gene regulation, RNA Biology, 15:12, 14331439, DOI: $10.1080 / 15476286.2018 .1551693$

48. Antisense \& RNAi Therapeutics Market Size, Share \& Trends Analysis Report By Route of Administration (Pulmonary Delivery, Intravenous Injection), By Application (CVDs, Oncology), By Technology, And Segment Forecasts, 2019 - 2025 Report ID: 978-1-68038-805-3

49. Hanna J, Hossain GS, Kocerha J. The Potential for microRNA Therapeutics and Clinical Research. Front Genet. 2019;10:478. Published 2019 May 16. doi:10.3389/fgene.2019.00478

50. Chakraborty C, Sharma AR, Sharma G, Doss CGP, Lee SS. Therapeutic miRNA and siRNA: Moving from Bench to Clinic as Next Generation Medicine. Mol Ther Nucleic Acids. 2017; 8:132-143. doi:10.1016/j. omtn.2017.06.005

51. Fay, E. J., \& Langlois, R. A. (2018). MicroRNA-Attenuated Virus Vaccines. Non-coding RNA, 4(4), 25. https://doi.org/10.3390/ ncrna4040025

52. Benitez, A. A., Spanko, L. A., Bouhaddou, M., Sachs, D., \& tenOever, B. R (2015). Engineered Mammalian RNAi Can Elicit Antiviral Protection that Negates the Requirement for the Interferon Response. Cell reports, 13(7), 1456-1466. https://doi.org/10.1016/j.celrep.2015.10.020

53. Stenvang J, Petri A, Lindow M, Obad S, Kauppinen S. Inhibition of microRNA function by antimiR oligonucleotides. Silence. 2012;3(1):1. Published 2012 Jan 9. doi:10.1186/1758-907X-3-1

54. Lam, J. K., Chow, M. Y., Zhang, Y., \& Leung, S. W. (2015). siRNA Versus miRNA as Therapeutics for Gene Silencing. Molecular therapy. Nucleic acids, 4(9), e252. https://doi.org/10.1038/mtna.2015.23

55. Kristen, A. V., Ajroud-Driss, S., Conceicao, I., Gorevic, P., Kyriakides, T., and Obici, L. (2018). Patisiran, an RNAi therapeutic for the treatment of hereditary transthyretin-mediated amyloidosis. Neurodegener Dis. Manag. 9, 5-23. doi: 10.2217/nmt-2018-0033

56. Yang, J. (2019). Patisiran for the treatment of hereditary transthyretinmediated amyloidosis. Expert. Rev. Clin. Pharmacol. 12, 95-99. doi: 10.1080/17512433.2019.1567326 
Aneja et al. Microbiology Discovery 2020,

57. Gallant-Behm CL, Piper J, Dickinson BA, Dalby CM, Pestano LA, Jackson AL. A synthetic microRNA-92a inhibitor (MRG-110) accelerates angiogenesis and wound healing in diabetic and nondiabetic wounds. Wound Repair Regen. 2018;26(4):311-323. doi:10.1111/ wrr.12660

58. Li G, Fan Y, Lai Y, et al. Coronavirus infections and immune responses. J Med Virol. 2020;92(4):424-432. doi:10.1002/jmv.25685

59. Sungnak, W., Huang, N., Bécavin, C. et al. SARS-CoV-2 entry factors are highly expressed in nasal epithelial cells together with innate immune genes. Nat Med 26, 681-687 (2020). https://doi.org/10.1038/s41591020-0868-6

60. Christoph Muus, et al. (2020). Integrated analyses of single-cell atlases reveal age, gender, and smoking status associations with cell typespecific expression of mediators of SARS-CoV-2 viral entry and highlights inflammatory programs in putative target cells. Posted on bioRxiv.org on April 20, 2020.10.1101/2020.04.19.049254.

61. Catanzaro, M., Fagiani, F., Racchi, M. et al. Immune response in COVID-19: addressing a pharmacological challenge by targeting pathways triggered by SARS-CoV-2. Sig Transduct Target Ther 5, 84 (2020). https:// doi.org/10.1038/s41392-020-0191-1

62. Huang, C. et al. Clinical features of patients infected with 2019 novel coronavirus in Wuhan, China. Lancet Lond. Engl. 395, 497-506 (2020).

63. Levanova, A., \& Poranen, M. M. (2018). RNA Interference as a Prospective Tool for the Control of Human Viral Infections. Frontiers in microbiology, 9, 2151. https://doi.org/10.3389/fmicb.2018.02151

64. Leon-Icaza, Stephen \& Zeng, Mingtao \& Rosas-Taraco, Adrian. (2019). microRNAs in viral acute respiratory infections: immune regulation, biomarkers, therapy, and vaccines. 1. 7. 10.1186/s41544-018-0004-7.

65. Mallick B, Ghosh Z, Chakrabarti J. MicroRNome analysis unravels the molecular basis of SARS infection in bronchoalveolar stem cells. PLoS One. 2009;4:e7837.

66. Martinez M. A. (2020). Compounds with Therapeutic Potential against Novel Respiratory 2019 Coronavirus. Antimicrobial agents and chemotherapy, 64(5), e00399-20. https://doi.org/10.1128/AAC.00399-20

67. Agostini, M. L., Andres, E. L., Sims, A. C., Graham, R. L., Sheahan, T. P., Lu, X., Denison, M. R. (2018). Coronavirus susceptibility to the antiviral remdesivir (GS-5734) is mediated by the viral polymerase and the proofreading exoribonuclease. MBio, 9(2). https://doi.org/10.1128/ mBio.00221-18

68. Marano, G., Vaglio, S., Pupella, S., Facco, G., Catalano, L., Liumbruno, G. M., \& Grazzini, G. (2016). Convalescent plasma: new evidence for an old therapeutic tool?. Blood transfusion = Trasfusione del sangue, 14(2), 152-157. https://doi.org/10.2450/2015.0131-15

69. Wu Y, Wang F, Shen $C$, et al. A noncompeting pair of human neutralizing antibodies block COVID-19 virus binding to its receptor ACE2 [published online ahead of print, 2020 May 13]. Science. 2020; eabc2241. doi:10.1126/science.abc2241

70. Wang, C., Li, W., Drabek, D. et al. A human monoclonal antibody blocking SARS-CoV-2 infection. Nat Commun 11, 2251 (2020). https://doi. org/10.1038/s41467-020-16256-y

71. Li J, Arévalo MT, Diaz-Arévalo D, Chen Y, Choi J-G, Zeng M. Generation of a safe and effective live viral vaccine by virus self-attenuation using species-specific artificial microRNA. J Control Release. 2015; 207:70-6.

72. Liu, C., Zhou, Q., Li, Y., Garner, L. V., Watkins, S. P., Carter, L. J., Smoot, J., Gregg, A. C., Daniels, A. D., Jervey, S., \& Albaiu, D. (2020). Research and Development on Therapeutic Agents and Vaccines for COVID-19 and Related Human Coronavirus Diseases. ACS central science, 6(3), 315-331. https://doi.org/10.1021/acscentsci.0c00272

73. Gruntman AM, Mueller C, Flotte TR, Gao G. Gene transfer in the lung using recombinant adeno-associated virus. Curr Protoc Microbiol. 2012 Aug;Chapter 14:Unit14D.2.

74. Abdel-Ghany, Shaimaa \& Sabit, Hussein. (2020). microRNA-Based Vaccination and Treatment for COVID-19. 4. 10.31021/ctvv.20203109.

75. Rational designing asymmetrical siRNAs to target Novel Acute Respiratory Syndrome RNA Coronavirus (COVID-19) for silencing (2020, IP Disclosure) Peixuan Guo (Ohio State University), Guiliang Tang (Michigan
Tech University), and Jianjun Zhao (Cleveland Clinic)

76. Methods and compositions for controlling efficacy of RNA silencing. (2019) Phillip D Zamore, Gu, Guiliang Tang US Patent 10,364,429

77. Zhang L, Hou D, Chen X, et al. Exogenous plant MIR168a specifically targets mammalian LDLRAP1: evidence of cross- -kingdom regulation by microRNA. Cell Res. 2012; 22: 107-126.

78. Zhou Z, Li X, Liu J, et al. Honeysuckle-encoded atypical microRNA2911 directly targets influenza A viruses. Cell Res. 2015;25:39-49.

79. Yang J, Farmer LM, Agyekum AA, et al. Detection of an abundant plantbased small RNA in healthy consumers. PLoS One. 2015;10:e0137516.

80. Sanchita, Trivedi R, Asif MH, Trivedi PK. Dietary plant miRNAs as an augmented therapy: cross-kingdom gene regulation. RNA Biol. 2018;15(12):1433-1439. doi:10.1080/15476286.2018.1551693

81. Lingbeck, J. M., O’Bryan, C. A., Martin, E. M., Adams, J. P., \& Crandall, P. G. (2015). Sweetgum: An ancient source of beneficial compounds with modern benefits. Pharmacognosy reviews, 9(17), 1-11. https://doi. org/10.4103/0973-7847.156307

82. Cavalieri, D., Rizzetto, L., Tocci, N. et al. Plant microRNAs as novel immunomodulatory agents. Sci Rep 6, 25761 (2016). https://doi. org/10.1038/srep25761

83. Liu T, Zhang L, Joo D, Sun SC. NF-kB signaling in inflammation. Signal Transduct Target Ther. 2017;2:17023-. doi:10.1038/sigtrans.2017.23

84. Şimşek Yavuz S, Ünal S. Antiviral treatment of COVID-19. Turk J Med Sci. 2020;50(SI-1):611-619. Published 2020 Apr 21. doi:10.3906/sag-2004145

85. Wang, X., Cao, R., Zhang, H. et al. The anti-influenza virus drug, arbidol is an efficient inhibitor of SARS-CoV-2 in vitro. Cell Discov 6, 28 (2020). https://doi.org/10.1038/s41421-020-0169-8

86. Hodgson, J. The pandemic pipeline. Nat Biotechnol. 2020;38(5):523-532. doi:10.1038/d41587-020-00005-z

87. Siomi, M. C. (2010). piRNAs (PIWI-interacting RNAs). AccessScience. Retrieved August 22, 2020, from https://doi.org/10.1036/1097-8542. YB100037

88. Anderson, E. J., Rouphael, N. G., Widge A. T., et al., (2020) Safety and Immunogenicity of SARS-CoV-2 mRNA-1273 Vaccine in Older Adults. $N$ Engl J Med. Sep 29

89. Walsh, E. E, Frenck, R. W. Jr, Falsey, A. R., et al. Safety and immunogenicity of two RNA-Based Covid-19 vaccine candidates. N Engl J Med. 2020 Oct 14:NEJMoa2027906. doi: 10.1056/NEJMoa2027906. Epub ahead of print. PMID: 33053279; PMCID: PMC7583697.

90. Keech C, Albert G, Cho I, Robertson A, et al. Phase 1-2 Trial of a SARSCoV-2 Recombinant Spike Protein Nanoparticle Vaccine. N Engl J Med. 2020 Sep 2:NEJMoa2026920. doi: 10.1056/NEJMoa2026920. Epub ahead of print. PMID: 32877576; PMCID: PMC7494251.

91. Mercado, N. B., Zahn, R., Wegmann, F. et al. Single-shot Ad26 vaccine protects against SARS-CoV-2 in rhesus macaques. Nature 586, 583-588 (2020). https://doi.org/10.1038/s41586-020-2607-z

92. Kalnin, K. V., Plitnik, T., Kishko, M, et al. Immunogenicity of novel mRNA COVID-19 vaccine MRT5500 in mice and 2 nonhuman primates. BioRxiv 2020.10.14.337535; doi: https://doi. org $/ 10.1101 / 2020.10 .14 .337535$

\section{Citation:}

Aneja KK, Dixit N and Kumar A. Can RNAi be used as a weapon against COVID-19/SARSCoV-2?. Microbiol Discov. 2020; 8:1.

http://dx.doi.org/10.7243/2052-6180-8-1 